\title{
CONJUNCTIVAL IMPRESSION CYTOLOGY IN DIAGNOSIS OF DRY EYE IN PRESENCE OF NORMAL TEAR FILM FUNCTION
}

\author{
Vandana Telgote1, Chandni Karole2, Pushpa Varma ${ }^{3}$, Priti Meshram 4
}

${ }^{1}$ Associate Professor, Department of Ophthalmology, Index Medical College Hospital and Research Centre, Indore. ${ }^{2}$ Assistant Professor, Department of Ophthalmology, Index Medical College Hospital and Research Centre, Indore. 3 Professor and HOD, Department of Ophthalmology, Sri Aurobindo Institute of Medical Sciences Hospital, Indore. ${ }_{4}^{4}$ PG Student, Department of Ophthalmology, M.G.M. Medical College Hospital, Indore.

\section{ABSTRACT}

\section{AIM}

To evaluate the ocular surface changes in patients with various conditions of dry eye with normal tear film function tests.

\section{METHODS}

20 cases of study group and 20 of control group are taken. Both groups underwent various tear film function tests including TBUT, Schirmer's test and rose Bengal staining and patients with abnormal tear film function tests were excluded from the study.

\section{RESULTS}

Statistical analysis was carried out using fisher's test with SPSS software. There was statistically significant difference between the patient and the control group in terms of impression cytology results (Fisher's test $\mathrm{p}=0.0037$ ).

1. In our study, $55 \%$ of case revealed grade zero conjunctival metaplasia, $25 \%$ showed grade $1,10 \%$ had grade 2 and rest $10 \%$ had grade 3 conjunctival metaplasia.

2. All the samples from control group had showed no conjunctival metaplasia.

3. Impression cytology result were found to be abnormal in $30 \%$ of NIDDM, $35 \%$ of allergic conjunctivitis and $35 \%$ of pterygium cases.

\section{CONCLUSIONS}

Impression cytology helps in early diagnosis of dry eye compared with commonly used tear film function tests, hence helpful in early establishment of treatment.

\section{PURPOSE}

To evaluate the ocular surface changes in patients with various conditions of dry eye with normal tear film function tests.

\section{KEYWORDS}

TBUT, Schirmer's test and rose Bengal Staining, Patients with Abnormal Tear Film Function Tests, Impression Cytology and Conjunctival Metaplasia.

HOW TO CITE THIS ARTICLE: Telgote V, Karole C, Varma P, et al. Conjunctival impression cytology in diagnosis of dry eye in presence of normal tear film function. J. Evolution Med. Dent. Sci. 2016;5(61):4272-4276, DOI: 10.14260/jemds/2016/975

\section{INTRODUCTION}

Impression cytology refers to the application of cellulose acetate filter paper to the ocular surface to remove the superficial layers of the ocular surface epithelium. The cells thus removed can be subjected to histological, immunohistological or molecular analysis. Egbert et al first described this minimally invasive method of studying conjunctival goblet cells in $1977 .^{1}$

It is non-invasive, easy to perform and yields reliable information about the area sampled with minimal discomfort to the patient. This makes it a valuable tool in the understanding of ocular surface disorders. Applications of impression cytology include the aetiological diagnosis of various ocular surface disorders, documenting sequential

Financial or Other, Competing Interest: None.

Submission 12-03-2016, Peer Review 12-05-2016,

Acceptance 19-05-2016, Published 30-07-2016.

Corresponding Author:

Dr. Chandni Karole,

\#363, At Post Khalwa

TQ-Khalwa Dist.,

Khandwa-450117,

Madhya Pradesh.

E-mail:dr.chandni777@gmail.com

DOI: $10.14260 /$ jemds $/ 2016 / 975$ changes in the conjunctival and corneal surface over the time, monitoring effects of treatment and staging conjunctival squamous metaplasia and as an investigational tool for analysing ocular surface disease with immune-staining and DNA analysis. ${ }^{2}$

Measurement of the tear breakup time is a very rough test for the determination of tear film stability and associated with large inter-individual and intra-individual deviations even when performed as a standardised procedure. As a systemic disease, diabetes mellitus affects the eyes in many ways along with typical dry eye symptoms, such as burning and/or foreign body sensation. Thus, the question arises as to whether diabetes mellitus is correlated with tear film anomalies or a disturbance of the function of the tear film.

Up till now, little information has been available on the function of the tear film in diabetic patients. In the present study we investigated the amount of tear production, the stability of the tear film and the condition of the conjunctival surface in non-insulin dependent diabetic individuals along with pts. of pterygium and allergic conjunctivitis.

\section{MATERIAL AND METHOD}

- $\quad$ Patients attending the O.P.D. of Ophthalmology Dept. of Index Medical College Hospital, Indore, between Aug. 2015 - Jan. 2016. 
- 20 cases of study group and 20 of control group are taken.

\section{STUDY GROUP}

20 Eyes of Various Dry Eye Conditions, out of which

- 7 Pterygium.

- 7 Allergic Conjunctivitis.

- 6 Diabetes Mellitus Type 2.

\section{METHODS}

A case control study including consecutive cases of dry eye syndrome was carried out. Individuals without dry eye were taken as control. Impression of conjunctiva with cellulose acetate filter paper was taken from inferonasal-bulbar conjunctiva and was stained with Periodic Acid-Schiff (PAS) and counter-stained with haematoxylin and eosin.

Patients with abnormal tear film function tests were excluded from the study.

\section{TESTS PERFORMED ON CASES AND CONTROLS TBUT - Tear Film Break Up Time}

TFBUT is the time period between a last blink and appearance of first dry spot after instilling one drop of fluorescein in conjunctival sac seen by slit lamp. This time is usually more than the time interval between two consecutive blinks (Holly and Lamp, 1977).

Normal TFBUT is from 15-34 seconds and if it is less than 10 seconds it is considered abnormal. Instability of tear film suggests mucin deficient dry eye or indicates lipid abnormality as shown by McCulley.

\section{Basal Schirmer's Test}

This test measures aqueous part of tear film. Tear production rate is seen by wetting of strip of filter paper, which is kept in lower conjunctival sac for 5 minutes. This test was devised by Schirmer in 1903. He measured the response of tear producing glands to a standard non-physiological stimulus by a filter paper. This response is called as lacrimation.

Schirmer's test is divided as Schirmer's test I and II. In Schirmer's test I, a $5 \times 35 \mathrm{~mm}$ strip of filter paper of which $5 \times 5$ $\mathrm{mm}$ is kept in lower conjunctival sac at junction of middle twothird and lateral one-third and the wetting was noted. In Schirmer's test II, nasal mucosa is irritated mechanically when the eye was anaesthetized.

According to Schirmer's, wetting of $30 \mathrm{~mm}$ of strip within 5 minutes should be present on an average in normal persons. Wetting less than $15 \mathrm{~mm}$ is pathological and wetting of whole strip within 20 seconds indicates hyper-secretion. When wetting is less than $15 \mathrm{~mm}$, then Schirmer's test II should be performed to rule out damage to excito-lacrimal fibres of lacrimal gland.

\section{Rose Bengal Staining}

Rose Bengal is a fluorescein derivative, which stains dead and damaged cells and mucus with no effect over epithelial defects or intercellular spaces. It is prepared as a $1 \%$ solution.

It causes only a mild discomfort to the patient except in the patients with dry eye, who are more uncomfortable. Therefore, it should be applied with cotton tipped applicator which is just touched to edges of dropper with Rose Bengal and then it is touched to tear lake.

Results were interpreted according to Von Bijster Veld (1969). He gave a score of 3 for each nasal and temporal conjunctiva in palpebral aperture and for cornea with a total score of 9 .

\section{Scoring was done as follows \\ 0 - Absent Stain. \\ 1 - Just Present. \\ 2 - Moderate Stain. \\ 3 - Gross stain.}

Sum of areas was done and score of more than 4 was taken as abnormal.

\section{Conjunctival Impression Cytology}

Generally, two to three layers of cells are removed in one application, but deeper cells can be accessed by repeat application over the same site. This is best achieved with a team approach including the ophthalmologist, pathologist, microbiologist and the immunologist.

\section{Specimen Collection}

The type of filter paper used and the technique of cell collection depend on the purpose for which the specimen is collected. The size of the filter paper pores affects the consistency of epithelial cells collected and the resolution of cell detail. Larger pore sizes collect cells better, but the cell detail is less well preserved. Treatment of the filter paper with surfactant also reduces cell pickup. Acetate cellulose filter paper strips with Pore size $0.20 \mu \mathrm{m}$ size and $13 \mathrm{~mm}$ diameter were used. ${ }^{3}$

After one drop of topical anaesthetic to each eye, wipe out excessive tear fluids and apply the filter paper to the desired area after Millipore paper divided into two " $\mathrm{D}$ "-shaped halves. The end of the paper to be applied to the nasal side is clipped or marked for orientation. Applying gentle pressure over the strip against the ocular surface, the paper is allowed to remain in contact with the eye for approximately 5-10 seconds.

Remove the filter paper by picking up the tip of the filter with the same forceps and follow a "Peeling" manoeuvre over the ocular surface. Drop the filter paper into one of the sample bottle (Vial), which contains the fixative solution and seal the bottle by screw. The sample is good for processing so long as the fixative does not dry out.

\section{Specimen Staining}

Haematoxylin and PAS stains are the commonly used stains for routine histological staining of impression cytology specimens. Staining is done in following steps.

1. The filter paper with the specimen is fixed for approximately 10 minutes in a solution containing glacial acetic acid, formaldehyde, and ethyl alcohol in a 1:1:20 volume ratio.

2. A 24 well culture plate or a 24 well Teflon sample holder is used to hold the specimens during fixation and staining.

3. The specimens are rehydrated in $70 \%$ ethyl alcohol and then placed successively in periodic acid Schiff reagent, sodium metasulfite, Gill's haematoxylin, and Scott's tap water substitute for 2 minutes each, rinsing in two changes of tap water in between each step. 4

4. This is followed by dehydration in two changes of $95 \%$ ethyl alcohol, staining with modified orange G for 2 minutes, rinsing in 95\% ethyl alcohol for 3 minutes and staining with modified eosin $Y$ for 2 minutes, again rinsing in 95\% ethyl alcohol for 5-10 minutes before dehydration in absolute alcohol for 5 minutes. 
Throughout the staining, the cell side of the filter paper must be completely soaked with staining solution.

For each de-staining or rinsing, the holder is either dipped 10 times or suspended in a large jar with continuous magnetic stirring so that there is no need for constant monitoring.

5. After the final de-staining step, xylene is used to make the filter paper transparent.

Before mounting, the filter paper is placed with the epithelial cells facing up. The completed slides are examined by light microscopy.

Special staining techniques have been devised for studying the specimens by electron microscopy. 5 and immunecytochemistry. 6,7

\section{Microscopy and Clinical Applications}

Impression cytology usually removes only 1-3 cell layers and does not yield the same information as a flat mount or crosssection preparation of the ocular surface. It is therefore ideal for studying the surface epithelium rather than the basal epithelium or the basement membrane (Fig. 1).

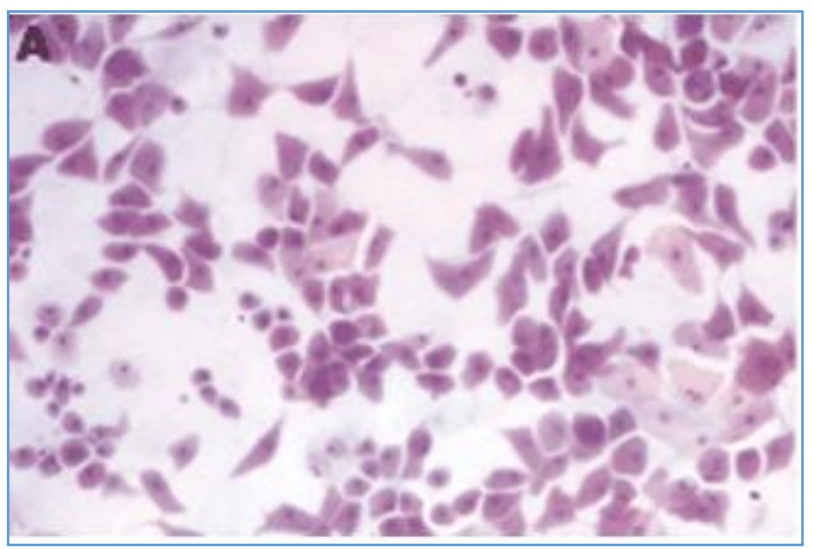

Fig. 1

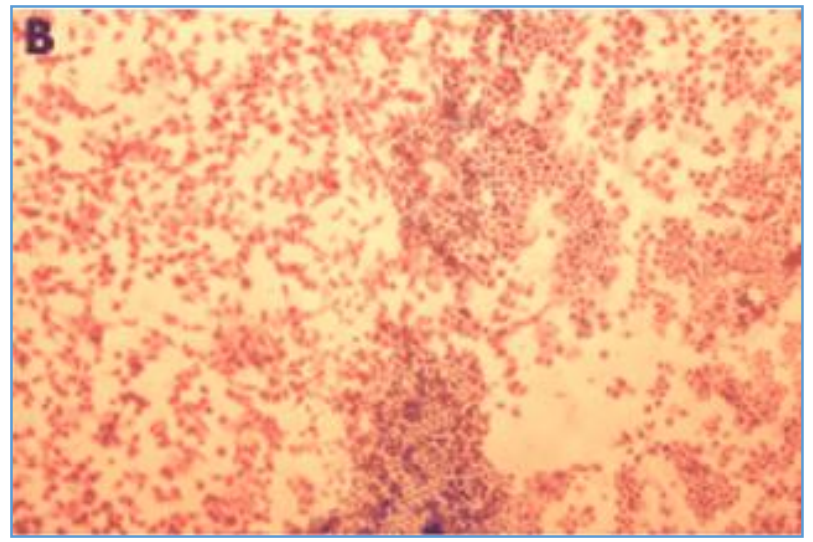

Fig. 2

A. Impression cytology of normal corneal surface showing corneal epithelial cells. Normal cells are flat with a prominent nucleus. The nuclear cytoplasmic ratio is low $(\times 100$, periodic acid Schiff staining).

B. Impression cytology of normal transition zone from cornea to limbus ( $\times 40$, periodic acid Schiff staining). The limbal epithelial cells are small, densely packed with a high nuclear cytoplasmic ratio. The limbal zone is clearly demarcated from the adjacent corneal epithelial cells.

1. In our study $55 \%$ of case revealed grade zero conjunctival metaplasia, $25 \%$ showed grade $1,10 \%$ had grade 2 and rest $10 \%$ had grade 3 conjunctival metaplasia.
Impression cytology has also been used in the evaluation of ocular surface diseases such as keratoconjunctivitis sicca, 8 vitamin A deficiency, ${ }^{9}$ cicatricial pemphigoid, ${ }^{10}$ atopic disease, ${ }^{11}$ superior limbic keratoconjunctivitis and mucopolysaccharidoses, vernal keratoconjunctivitis, 12 and the effect on these of various therapies.

Nelson graded conjunctival impression cytology specimens (Grades $0-3$, Table 1) based on the appearance of the epithelial cells and the numbers of goblet cells. ${ }^{13}$

\begin{tabular}{|c|c|}
\hline \multirow{2}{*}{ Grade 0} & $>500$ goblet cells $/ \mathrm{mm}^{2}$ \\
\cline { 2 - 3 } & Small, round epithelial cells with large nuclei \\
\hline Grade 1 & Goblet cells: $350-500$ cells $/ \mathrm{mm}^{2}$ \\
\hline Grade 2 & Goblet cells: $100-150$ cells $\mathrm{mm}^{2}$ \\
\hline Grade 3 & $\begin{array}{c}<100 \text { goblet cells } / \mathrm{mm}^{2} \\
\text { Large, polygonal epithelial cells with small } \\
\text { nuclei }\end{array}$ \\
\hline \multicolumn{2}{|c|}{ Table 1: Nelson's Classification for Squamous } \\
Metaplasia.14
\end{tabular}

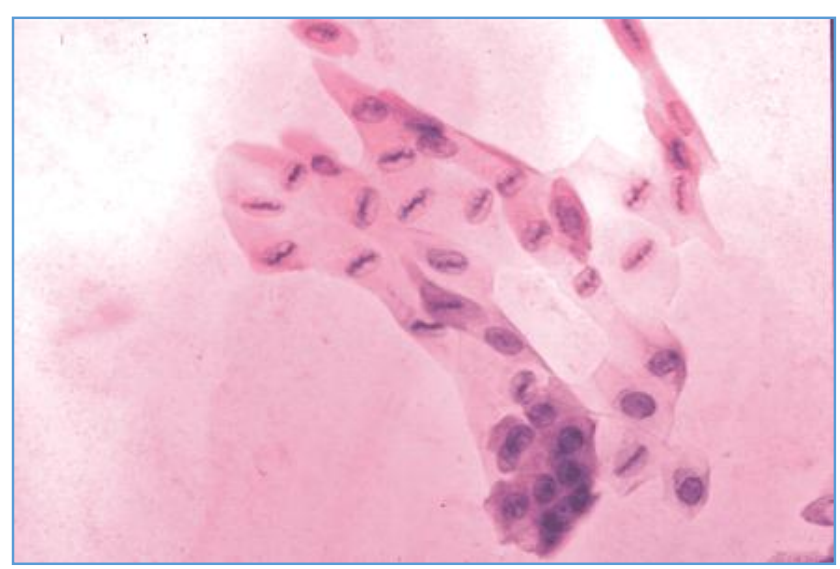

Fig. 3

Impression cytology of the conjunctival surface showing snake-like chromatin in keratoconjunctivitis sicca $(\times 100$, periodic acid Schiff staining).

While there are numerous clinical and research applications of impression cytology, it has not yet become a routine diagnostic tool in most clinics, because it is relatively cumbersome and time consuming for both the clinician and pathologist. However, the ability to obtain multiple samples of the ocular surface at one sitting with minimal discomfort to the patient makes it an ideal method of investigating ocular surface disorders when the diagnosis is not clinically obvious or when the clinical diagnosis needs to be substantiated and documented. It is also a handy research tool. We recommend that major ophthalmic centres should develop and introduce this technique into routine clinical practice. For this to be achieved a team approach including the ophthalmologist, pathologist, microbiologist and the immunologist is essential.

\section{OBSERVATIONS}

Statistical analysis was carried out using Fisher's test with SPSS software. 


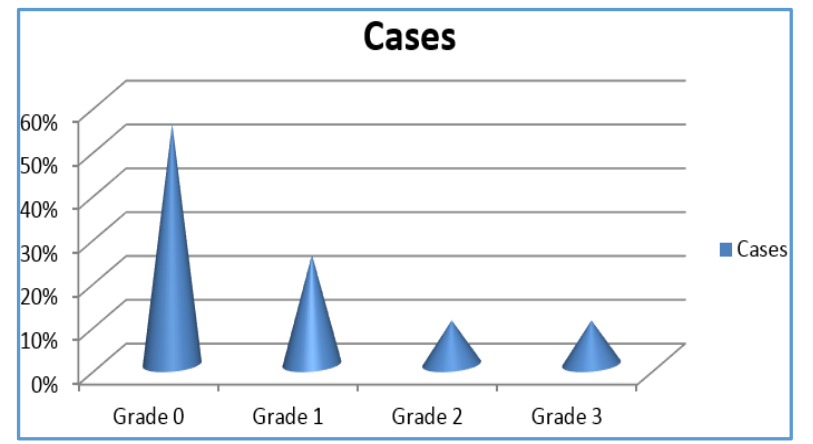

2. All the samples from control group had showed no conjunctival metaplasia.

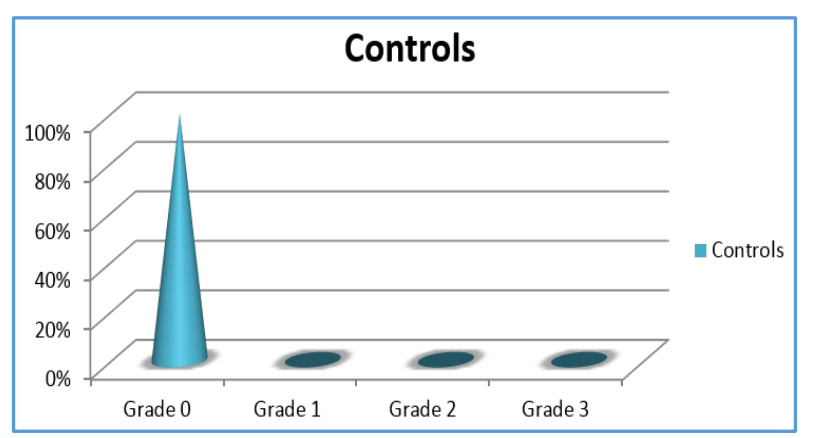

3. Impression cytology results were found to be abnormal in $30 \%$ of NIDDM, $35 \%$ of allergic conjunctivitis and $35 \%$ of pterygium cases.

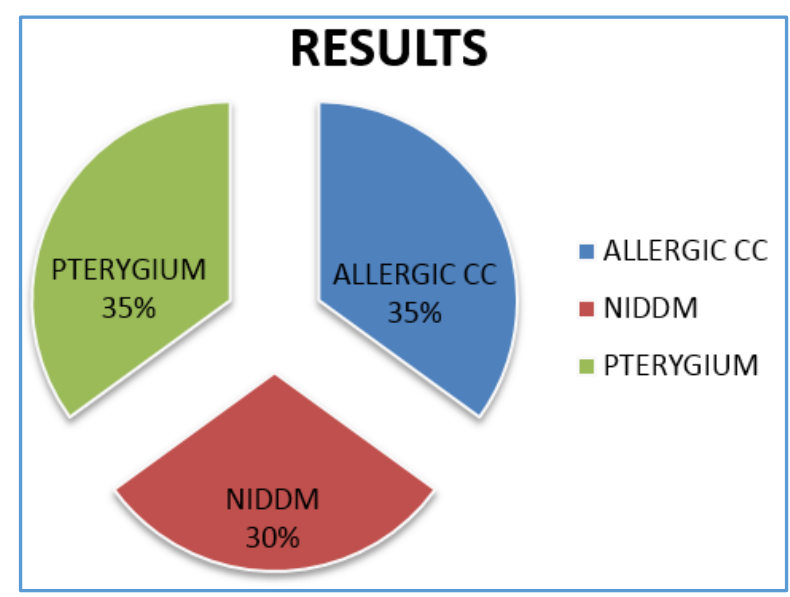

4. Out of 7 allergic conjunctivitis eyes, only $28 \%$ eyes showed squamous metaplasia and $71 \%$ were normal.

5. Out of 6 NIDDM eyes 50\% were normal and 50\% results showed squamous metaplasia.

6. Out of 7 pterygium eyes, $57 \%$ had squamous metaplasia of grade 1 and 2 , and $42 \%$ were normal.

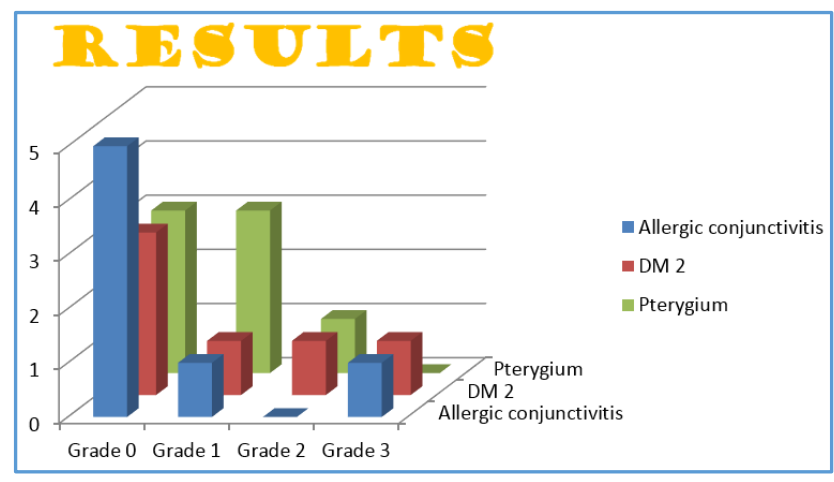

This graph shows comparison of Grades of squamous metaplasia in Pterygium cases, NIDDM cases and allergic conjunctivitis cases.

\section{RESULTS}

There was statistically significant difference between the patient and the control group in terms of impression cytology results (Fisher's test $\mathrm{p}=0.0037$ ).

\section{DISCUSSION}

Impression cytology of the conjunctiva is an important diagnostic tool in evaluation of ocular surface disorders with abnormal tear film function tests such as

- Xerophthalmia.

- Ocular pemphigoid.

- Keratoconjunctivitis sicca.

- Stevens-Johnson syndrome.

In our study, we have extended the use of impression cytology to evaluate the ocular surface morphology in ocular surface disorders with normal tear film function tests such as

- $\quad$ Allergic conjunctivitis.

- $\quad$ Type 2 diabetes mellitus.

- Pterygium.

Martin Goebbel (2000) has studied 86 eyes of non-insulin dependent DM for TBUT, Schirmer's and impression cytology. 15

They found no significant difference in terms of results of TBUT between study and control population.

However, statistically significant difference was found between two populations in terms of impression cytology results.

In our study out of 6 patients of NIDDM with normal tear film function tests, 3 patients had abnormal results of impression cytology.

Dr. Usha Yadava, Dr. Pankaj Sachdeva, Dr. J.L.G 2005 has studied conjunctival surface using impression cytology in 40 eyes with vernal conjunctivitis symptomatic for 14-32 days and compared them with age matched controls. ${ }^{16}$

They concluded that tear film function tests give only indirect knowledge about the modifications occurring in the epithelium and thus impression cytology provides a good compromise between clinical and histological investigations. 
In our study, 2 eyes of 7 patients of allergic conjunctivitis disclosed abnormal results of impression cytology in presence of normal tear film function tests, which was statistically significant. Ahmet Ergin, Önder Bozdoan 2001 have evaluated tear film function in 86 eyes of Pterygium and compared them with same number of controls. ${ }^{17}$

Statistically no significant difference was found between cases and controls in this study suggesting the unreliability of these tests in case of Pterygium.

In present study, 4 of 7 patients of Pterygium with normal tear film function tests are positive for impression cytology, suggesting its role in determination of dry eye.

\section{CONCLUSION}

- Most of the studies have evaluated the ocular surface changes using both tear film function tests and impression cytology showing positive results in term of both tear film function tests and impression cytology.

- Our study strongly favours the use of impression cytology as a single novel approach in evaluation of ocular surface changes.

- $\quad$ Also, we conclude that impression cytology helps in early diagnosis of dry eye compared with commonly used tear film function tests, hence helpful in early establishment of treatment.

\section{REFERENCES}

1. Egbert PR, Lauber S, Maurice DM. A simple conjunctival biopsy. Am J Ophthalmol 1977;84(6):798-801.

2. Tseng SCG. Staging of conjunctival squamous metaplasia by impression cytology. Ophthalmology 1985;92(6):728-33.

3. Vadrevu VLD, Fullard RJ. Enhancements to the conjunctival impression cytology technique and examples of applications in a clinico-biochemical study of dry eye. CLAO J 1994;20(1):59-63.

4. Gill GW, Frost JK, Miller KA. A new formula for a halfoxidised haematoxylin solution that neither over stains nor requires differentiation. Acta Cytol 1974;18(4):300-11.
5. Maskin SL, Bodé DD. Electron microscopy of impressionacquired conjunctival epithelial cells. Ophthalmology 1986;93(12):1518-23.

6. Krenzer KL, Freddo TF. Cytokeratin expression in normal human bulbar conjunctiva obtained by impression cytology. Invest Ophthalmol Vis Sci 1997;38(1):142-52.

7. Thiel MA, Bossart W, Bernauer W. Improved impression cytology techniques for the immunopathological diagnosis of superficial viral infections. $\mathrm{Br} \mathrm{J}$ Ophthalmol 1997;81(11):984-8.

8. Marner K. Snake-like appearance of nuclear chromatin in conjunctival epithelial cells from patients with keratoconjunctivitis sicca. Acta Ophthalmol (Copenh) 1980;58(5):849-53.

9. Nelson JD, Wright JC. Conjunctival goblet cell densities in ocular surface disease. Arch Ophthalmol 1984;102(7):1049-51.

10. Wittpenn JR, Tseng SC, Sommer A. Detection of early xerophthalmia by impression cytology. Arch Ophthalmol 1996;104(2):237-9.

11. Nelson JD. Ocular surface impression using a cellulose acetate filter material. Arch Ophthalmol 1982;27:67-9.

12. Dogru M, Katakami C, Nakagawa N, et al. Impression cytology in atopic dermatitis. Ophthalmology 1998;105(8):1478-84.

13. Aragona P, Romeo G, Puzzolo D, et al. Impression cytology of the conjunctival epithelium in patients with vernal conjunctivitis. Eye 1996;10(Pt 1):82-5.

14. Nelson DJ. Impression cytology. Cornea 1988;7(1):71-81.

15. Martin Goebbel, Tear Secretion And Tear Film Function In Insulin Dependent Diabetics Bjo Jan 2000;84(1):19-21

16. Yadava U, sachdeva P, Goyal, JL. Conjunctival impression cytology: a control study in patients with vernal conjunctivitis. All India Ophthal Conf 2005;577-8.

17. Ahmet Ergin, Önder Bozdoan. Study on tear function abnormality in pterygium. Ophthalmologica 2001;215(3):204-208. 